\title{
LARGE DEVIATION PROBABILITIES FOR SUMS OF HEAVY-TAILED DEPENDENT RANDOM VECTORS*
}

\section{Adam Jakubowski Alexander V. Nagaev \\ Alexander Zaigraev}

Nicholas Copernicus University

Faculty of Mathematics and Computer Science

ul. Chopina 12/18, 87-100 Torun, Poland

E-mail: adjakubo@-nagaev@-alzaig@mat.uni.torun.pl

\begin{abstract}
Necessary and sufficient conditions are given for multidimensional $p$ - stable limit theorems (i.e. theorems on convergence of normalized partial sums $S_{n} / b_{n}$ of a stationary sequence of random vectors to a non-degenerate strictly $p$-stable limiting law $\mu$, with $1 / p$ regularly varying normalizing sequence $b_{n}$ ). It is proved that similarly as in the one-dimensional case the conditions for $0<p<2$ consist of two parts: one responsible for (very weak) mixing properties and another, describing asymptotics of probabilities of large deviations (with a minor additional condition for $p=1$ ). The paper focuses on effective methods of proving such large deviation results.
\end{abstract}

Key Words And Phrases: multivariate stable distributions, regular variation, stable limit theorems, large deviations, stationary sequences, $\psi$-mixing, $\phi$-mixing, $m$-dependence.

* Supported by Komitet Badań Naukowych under Grant PB 591/P03/95/08 


\section{A MULTIDIMENSIONAL STABLE LIMIT THEO- REM}

Let $X_{1}, X_{2}, \ldots$ be a stationary sequence of $d$-dimensional random vectors with partial sums $S_{0}=0, S_{n}=\sum_{j=1}^{n} X_{j}$. Following Jakubowski (1993) we will say that a $p$-stable limit theorem holds for $\left\{X_{j}\right\}$ if there exist a non-degenerate strictly $p$-stable law $\mu$ on $\mathbb{R}^{d}$ and a $1 / p$-regularly varying sequence $b_{n}$, such that

$$
\frac{S_{n}}{b_{n}} \underset{\mathcal{D}}{\longrightarrow} \mu, \quad \text { as } n \rightarrow+\infty .
$$

Recall that a $p$-stable law $\mu$ is strictly stable if for all $a, b>0$ one can find $c=c(a, b)>0$ such that $\left(\mu \circ R_{a}^{-1}\right) *\left(\mu \circ R_{b}^{-1}\right)=\mu \circ R_{c}^{-1}$, where for $a>0, R_{a}(x)=a \cdot x$ is a rescaling of $\mathbb{R}^{d}$. For the case $p \neq 1,2$, the $\operatorname{logarithm} \log \hat{\mu}(y)$ of the characteristic function of a strictly $p$-stable law can be written in the form

$$
\eta_{p} \int_{\left\{s \in S^{d-1} ;\langle y, s\rangle>0\right\}}|\langle y, s\rangle|^{p} \kappa(d s)+\overline{\eta_{p}} \int_{\left\{s \in S^{d-1} ;\langle y, s\rangle<0\right\}}|\langle y, s\rangle|^{p} \kappa(d s),
$$

where $y \in \mathbb{R}^{d}, S^{d-1}$ is the $d$-1-dimensional unit sphere in $\mathbb{R}^{d}$, $\kappa$ is a finite Borel measure on $S^{d-1}$ and

$$
\eta_{p}= \begin{cases}\int_{0}^{\infty}\left(e^{i u}-1\right) u^{-(1+p)} d u & \text { if } 0<p<1, \\ \int_{0}^{\infty}\left(e^{i u}-1-i u\right) u^{-(1+p)} d u & \text { if } 1<p<2 .\end{cases}
$$

For $p=1$, a law $\mu$ is strictly stable if it is a shift of a symmetric strictly stable law with logarithm of the characteristic function of the form

$$
-(1 / 2) \pi \int_{S^{d-1}}|\langle y, s\rangle| \kappa(d s),
$$

where $\kappa$ is a symmetric measure on $S^{d-1}$. Let us denote by Stab $(p, \kappa)$ the strictly stable law with logarithm of the characteristic function given by formulas (2) or (4). For more information on stable laws and processes we refer to Samorodnitsky and Taqqu (1994). 
For random variables $(d=1)$ Jakubowski $(1993,1997)$ obtained necessary and sufficient conditions for a $p$-stable limit theorem to hold. In the case of heavy-tailed random variables (i.e. if $0<p<2$ ) the conditions essentially consist of two parts: a part responsible for "mixing" properties (Condition $\mathrm{B}_{1}$ below) and a part describing asymptotic behaviour of probabilities of large deviations (Condition $\mathrm{LD}_{1}$ below). In both cases subscript 1 stands for the dimension $d=1$. Formal statements are as follows.

- Condition $\mathbf{B}_{1}$. For each $\lambda \in \mathbb{R}^{1}$, and as $n \rightarrow+\infty$

$$
\max _{\substack{1 \leq k, l \leq n \\ k+l \leq n}}\left|E e^{i \lambda\left(S_{k+l} / b_{n}\right)}-E e^{i \lambda\left(S_{k} / b_{n}\right)} \cdot E e^{i \lambda\left(S_{l} / b_{n}\right)}\right| \longrightarrow 0 .
$$

- Condition $\mathbf{L D}_{1}$. There exists a sequence $r_{n} \rightarrow+\infty$ such that for all sequences $x_{n}$ increasing to $+\infty$ slowly enough (i.e. $x_{n}=o\left(r_{n}\right)$ )

$$
x_{n}^{p} P\left(S_{n} / b_{n}>x_{n}\right) \longrightarrow c_{+}, \quad x_{n}^{p} P\left(S_{n} / b_{n}<-x_{n}\right) \longrightarrow c_{-},
$$

where $0<c_{+}+c_{-}<+\infty$ and $b_{n} \rightarrow+\infty$.

The relations between Conditions $\mathrm{B}_{1}$ and $\mathrm{LD}_{1}$ and $p$-stable limit theorems are particularly appealing in the case $p \neq 1$, as the following theorem shows (see Jakubowski, 1993 for the case $0<p<1$ and 1997 for the case $1<p<2$ ).

Theorem 1 Let $0<p<1$ or $1<p<2$. Suppose Conditions $B_{1}$ and $L D_{1}$ hold with $b_{n} \rightarrow+\infty$ and $0<c_{+}+c_{-}<+\infty$. Then $b_{n}$ varies $1 / p$-regularly and as $n \rightarrow+\infty$

$$
\frac{S_{n}}{b_{n}} \underset{\mathcal{D}}{\longrightarrow} \operatorname{Stab}\left(p, \kappa_{\left(c_{+}, c_{-}\right)}\right),
$$

where $\kappa_{\left(c_{+}, c_{-}\right)}\{+1\}=c_{+}$and $\kappa_{\left(c_{+}, c_{-}\right)}\{-1\}=c_{-}$.

Conversely, (7) with $c_{+}+c_{-}>0$ and $1 / p$-regular variation of $b_{n}$ imply Conditions $B_{1}$ and $L D_{1}$. 
Notice that for $d=1$ we have $S^{d-1}=S^{0}=\{-1,+1\}$.

For $p=1$ a minor additional assumption on centering is necessary (see Theorem 2.2, Jakubowski, 1997), which is automatically satisfied, when the $S_{n}$ 's are symmetric:

Theorem 2 If $p=1$ and for each $n \in \mathbb{N}$, the law of $S_{n}$ is symmetric, then Condition $B_{1}$ and Condition $L D_{1}$ with $0<c_{+}=$ $c_{-}=c<+\infty$ hold if, and only if,

$$
\frac{S_{n}}{b_{n}} \underset{\mathcal{D}}{\longrightarrow} \operatorname{Stab}\left(1, \kappa_{(c, c)}\right), \quad \text { as } n \rightarrow+\infty,
$$

where $0<c<+\infty$ and $b_{n}$ is regularly varying with exponent 1 .

The purpose of the present note is to prove a multidimensional generalization of the above results.

Let us begin with introducing a multidimensional version of Condition $\mathrm{B}_{1}$.

- Condition $\mathbf{B}_{d}$. For each $y \in \mathbb{R}^{d}$, and as $n \rightarrow+\infty$

$$
\max _{\substack{1 \leq k, l \leq n \\ k+l \leq n}}\left|E e^{i\left\langle y, S_{k+l} / b_{n}\right\rangle}-E e^{i\left\langle y, S_{k} / b_{n}\right\rangle} \cdot E e^{i\left\langle y, S_{l} / b_{n}\right\rangle}\right| \longrightarrow 0 .
$$

Condition $B_{d}$ describes a kind of "asymptotic independence" of partial sums. It is however essentially weaker than mixing conditions (such as $\alpha$-mixing) usually considered in limit theory for sums, for there exist non-ergodic sequences satisfying (9). On the other hand Condition $\mathrm{B}_{d}$ held for each $y \in \mathbb{R}^{d}$ implies (under mild additional assumptions) uniform convergence over bounded subsets of $\mathbb{R}^{d}$ :

$$
\max _{\substack{1 \leq k, l \leq n \\ k+l \leq n}} \sup _{\|y\| \leq K}\left|E e^{i\left\langle y, S_{k+l} / b_{n}\right\rangle}-E e^{i\left\langle y, S_{k} / b_{n}\right\rangle} \cdot E e^{i\left\langle y, S_{l} / b_{n}\right\rangle}\right| \longrightarrow 0,
$$

for every $K>0$ and as $n \rightarrow+\infty$. In particular, (10) implies that given Condition $\mathrm{B}_{d}$ for some normalizing sequence $\left\{b_{n}\right\}$, we obtain it for all sequences $b_{n}^{\prime}$ such that $b_{n} \leq C b_{n}^{\prime}, n \in \mathbb{N}$, for some 
constant $C>0$. This has been observed by Szewczak (1996). For examples of sequences satisfying Condition $\mathrm{B}_{d}$ and further discussion in the case $d=1$ (which can be easily extended to several dimensions) we refer to Jakubowski $(1991,1993)$.

The form of Condition $\mathrm{LD}_{d}$ is somewhat more complicated than (6) and involves convergence to a measure which is, in general, finite only outside of every neighborhood of $0 \in \mathbb{R}^{d}$ (hence $\sigma$-finite). In our theorems such measures will always be Lévy measures, but from the point of view of sufficiency of Condition $\mathrm{LD}_{d}$ it is reasonable to formulate this condition in full generality.

For further purposes, let us denote by $\nu(p, \kappa)$ the Lévy measure of the infinite divisible law $\operatorname{Stab}(p, \kappa)$. This means that for "radial" sets $A$ of the form $A=\cup_{x \in B} x \cdot V$, where $B \in \mathcal{B}_{\mathbb{R}^{+}}$and $V \in \mathcal{B}_{S^{d-1}}$, we have

$$
\nu(p, \kappa)(A)=\int_{B} u^{-1-p} d u \cdot \kappa(V) .
$$

Clearly, $\nu(p, \kappa)=0$ if, and only if, $\kappa=0$ and $\nu(p, \kappa)$ is symmetric if, and only if, $\kappa$ is symmetric.

- Condition $\mathbf{L D}_{d}$. There exists a sequence $b_{n} \rightarrow+\infty$ and a measure $\nu$ on $\mathbb{R}^{d}$, finite outside of every neighborhood of $0 \in$ $\mathbb{R}^{d}$, such that for all sequences $x_{n} \rightarrow+\infty$ increasing "slowly enough" (i.e. $x_{n}=o\left(r_{n}\right)$ for some sequence $r_{n} \rightarrow+\infty$ ) we have

$$
x_{n}^{p} P\left(S_{n} / b_{n} \in x_{n} A\right) \longrightarrow \nu(A),
$$

whenever $A \in \mathcal{B}^{d}, \bar{A} \not \supset 0$ and $\nu(\partial A)=0$.

Given Conditions $\mathrm{B}_{d}$ and $\mathrm{LD}_{d}$ we have a complete generalization of Theorems 1 and 2 .

Theorem 3 Let $0<p<1$ or $1<p<2$. Suppose Conditions $B_{d}$ and $L D_{d}$ hold with $b_{n} \rightarrow+\infty$ and $\nu \neq 0$.

Then $b_{n}$ varies $1 / p$-regularly, $\nu=\nu(p, \kappa)$ for some $\kappa \neq 0$ and

$$
\frac{S_{n}}{b_{n}} \underset{\mathcal{D}}{\longrightarrow} \operatorname{Stab}(p, \kappa), \quad \text { as } n \rightarrow+\infty .
$$


Conversely, (13) with $\kappa \neq 0$ and $1 / p$-regular variation of $b_{n}$ imply Conditions $B_{d}$ and $L D_{d}$ with $\nu=\nu(p, \kappa)$.

Theorem 4 Let $p=1$. Suppose for each $n \in \mathbb{N}$, the law of $S_{n}$ is symmetric. Then Condition $B_{d}$ and Condition $L D_{d}$ with symmetric $\nu=\nu(1, \kappa) \neq 0$ hold if, and only if,

$$
\frac{S_{n}}{b_{n}} \underset{\mathcal{D}}{\longrightarrow} \operatorname{Stab}(1, \kappa), \quad \text { as } n \rightarrow+\infty,
$$

where $\kappa \neq 0$ is symmetric and $b_{n}$ is regularly varying with exponent 1 .

Proof. Necessity of Condition $\mathrm{LD}_{d}$. In order to prove Condition $\mathrm{LD}_{d}$ we shall proceed similarly as in the one-dimensional case.

Let for each $n, Y_{n, 1}, Y_{n, 2}, \ldots$ be independent copies of $S_{n} / b_{n}$. By strict stability of $\mu$,

$$
k^{-1 / p} \sum_{j=1}^{k} Y_{n, j} \quad \underset{\mathcal{D}}{\longrightarrow} \mu, \quad \text { as } n \rightarrow+\infty, \quad k=1,2, \ldots
$$

It follows that there exists $r_{n} \nearrow+\infty$ such, that for every sequence $\left\{k_{n}\right\} \subset \mathbb{N}$, which is increasing to infinity slowly enough, i.e., $k_{n} \rightarrow+\infty, k_{n}=o\left(r_{n}\right)$, we have

$$
k_{n}^{-1 / p} \sum_{j=1}^{k_{n}} Y_{n, j} \underset{\mathcal{D}}{\longrightarrow} \mu, \quad \text { as } n \rightarrow+\infty .
$$

(Notice that condition (16) is considerably weaker than condition (15)).

Since $k_{n} \rightarrow \infty$, the array $\left\{k_{n}^{-1 / p} Y_{n, j}\right\}$ of row-wise independent random variables is infinitesimal and we can apply a convergence criterion for stable laws for sums of independent random variables. In particular, for each Borel subset $A \in \mathcal{B}^{d}$ which is separated from zero and such that $\nu(\partial A)=0$, we have as $n \rightarrow+\infty$

$$
k_{n} P\left(S_{n} /\left(b_{n} k_{n}^{1 / p}\right) \in A\right)=k_{n} P\left(S_{n} / b_{n} \in k_{n}^{1 / p} \cdot A\right) \longrightarrow \nu(A),
$$


where $\nu=\nu(p, \kappa)$ is the Lévy measure of the stable law $\mu$.

Setting $x_{n}=k_{n}^{1 / p}$ we obtain Condition $\mathrm{LD}_{d}$ with sequences $x_{n}$ of specific form and with rate $r_{n}^{1 / p}$. Due to the special form of the Lévy measure $\nu(p, \kappa)$ we can extend (17) to all sequences $x_{n} \rightarrow+\infty, x_{n}=o\left(r_{n}^{1 / p}\right)$.

Necessity of Condition $\mathrm{B}_{d}$. Let $y \in \mathbb{R}^{d}$. Then

$$
\left\langle y, S_{n} / b_{n}\right\rangle \underset{\mathcal{D}}{\longrightarrow} \mu_{y}
$$

where $\mu_{y}$ is the strictly stable law on $\mathbb{R}^{d}$ being an image of $\mu$ under the mapping $\mathbb{R}^{d} \ni x \mapsto\langle y, x\rangle \in \mathbb{R}^{1}$. If $y \in \mathbb{R}^{d}$ is such that $\mu_{y}$ is different from $\delta_{0}$, we obtain (9) from the corresponding theorem for $d=1$. If $\mu_{y}=\delta_{0}$, we have for any sequence $k_{n} \leq n$

$$
\left\langle y, \frac{S_{k_{n}}}{b_{n}}\right\rangle=\frac{b_{k_{n}}}{b_{n}} \cdot\left\langle y, \frac{S_{k_{n}}}{b_{k_{n}}}\right\rangle \underset{\mathcal{P}}{\longrightarrow} 0
$$

for if $k_{n^{\prime}} \rightarrow \infty$ along some subsequence $n^{\prime}$, then $\sup _{n} b_{k_{n}} / b_{n}<+\infty$ by regular variation of $b_{n}$ and if $k_{n^{\prime \prime}}$ remains bounded along some subsequence $n^{\prime \prime}$, then we have $b_{k_{n^{\prime \prime}}} / b_{n^{\prime \prime}} \rightarrow 0$ by $b_{n} \rightarrow \infty$. Hence if $k_{n}+l_{n} \leq n$, then $S_{k_{n}+l_{n}} / b_{n} \longrightarrow \mathcal{P} 0, S_{k_{n}} / b_{n} \longrightarrow \mathcal{P} 0$ and $S_{l_{n}} / b_{n} \longrightarrow \mathcal{P} 0$ and (9) is satisfied for $y$, too.

Sufficiency. Suppose Conditions $\mathrm{B}_{d}$ and $\mathrm{LD}_{d}$ hold for some $b_{n} \rightarrow \infty$ and some measure $\nu$ which is finite outside of every neighborhood of $0 \in \mathbb{R}^{d}$. Since strict stability of $\mu$ is equivalent to $\mu^{* n}=\mu \circ R_{n^{1 / p}}^{-1}$ for each $n \in \mathbb{N}$, it is sufficient to prove that for each $y \in \mathbb{R}^{d}$ one dimensional sums $\sum_{k=1}^{n}\left\langle y, X_{k} / b_{n}\right\rangle$ converge to some strictly $p$-stable law on $\mathbb{R}^{1}$ (possibly degenerated at 0 ).

Let us fix $y \in \mathbb{R}^{d}, y \neq 0$, and consider in (12) the following $\operatorname{sets} A_{+}^{y}, A_{-}^{y} \subset \mathbb{R}^{d}$

$$
A_{+}^{y}=\{x ;\langle y, x\rangle>1\}, \quad A_{-}^{y}=\{x ;\langle y, x\rangle<-1\} .
$$

These sets are separated from zero and we may assume that $\nu\left(\partial A_{ \pm}^{y}\right)=0$ (otherwise we may replace $y$ with $r \cdot y$ for some 
$1>r>0$ ). Moreover, by (12) we have

$x_{n}^{p} P\left(\sum_{k=1}^{n}\left\langle y, X_{k} / b_{n}\right\rangle>x_{n}\right)=x_{n}^{p} P\left(S_{n} / b_{n} \in x_{n} A_{+}^{y}\right) \rightarrow c_{+}=\nu\left(A_{+}^{y}\right)$,

provided $x_{n} \rightarrow+\infty$ slowly enough. Similar relation holds for the left-hand tails of $\sum_{k=1}^{n}\left\langle y, X_{k} / b_{n}\right\rangle$. It follows that in the case

$$
\nu\left(A_{+}^{y}\right)+\nu\left(A_{-}^{y}\right)>0
$$

we may apply either Theorem 1 (for $0<p<1$ and $1<p<2$ ) or Theorem 2 (for $p=1$ ) in order to get the convergence of $\left\{\sum_{k=1}^{n}\left\langle y, X_{k} / b_{n}\right\rangle\right\}$ to a non-degenerate strictly $p$-stable law. In particular, $b_{n}$ is $p$-regularly varying for there are $y$ 's satisfying (19) (by $\nu \neq 0$ ).

It remains to prove that regular $p$-variation of $b_{n}$ and $\nu\left(A_{+}\right)+$ $\nu\left(A_{-}\right)=0$ imply

$$
\sum_{k=1}^{n}\left\langle y, X_{k} / b_{n}\right\rangle \underset{\mathcal{P}}{\longrightarrow} 0
$$

This can be done in various ways. One can use, for example the normal convergence criterion (with the limit $\delta_{0}=\mathcal{N}(0,0)$ ) developed in Jakubowski and Szewczak (1991) together with the estimates of truncated moments given in Denker and Jakubowski (1989). Less formal is the following procedure. Take $\left\{Y_{k}^{c}\right\}$ to be independent, identically distributed and such that

$$
\sum_{k=1}^{n} Y_{k}^{c} / b_{n} \underset{\mathcal{D}}{\longrightarrow} \operatorname{Stab}\left(p, \kappa_{(c, c)}\right),
$$

where $\kappa_{(c, c)}$ is the same as in (7). By the corresponding onedimensional theorem, we have for $x_{n}$ increasing slowly enough

$$
x_{n}^{p} P\left(\sum_{k=1}^{n} Y_{k}^{c} / b_{n}>x_{n}\right) \rightarrow c,
$$

as well as

$$
x_{n}^{p} P\left(\sum_{k=1}^{n} Y_{k}^{c} / b_{n}<-x_{n}\right) \rightarrow c .
$$


It is now a matter of simple manipulations to deduce that we have also

$$
x_{n}^{p} P\left(\sum_{k=1}^{n}\left(Y_{k}^{c}+\left\langle y, X_{k}\right\rangle\right) / b_{n}>x_{n}\right) \rightarrow c,
$$

(and similarly for the left-hand tails). Since Condition $\mathrm{B}_{1}$ is obviously satisfied for the sequence $\left\{Y_{k}^{c}+\left\langle y, X_{k}\right\rangle\right\}$, we obtain

$$
\sum_{k=1}^{n}\left(Y_{k}^{c}+\left\langle y, X_{k}\right\rangle\right) / b_{n} \underset{\mathcal{D}}{\longrightarrow} \operatorname{Stab}\left(p, \kappa_{(c, c)}\right) \text {. }
$$

Letting $c \searrow 0$ we obtain (20).

\section{PROBABILITIES OF LARGE DEVIATIONS IN $\mathbb{R}^{d}$}

It follows from the proof of Theorems 3 and 4 that instead of Condition $\mathrm{LD}_{d}$ as it stands in (12) one can restrict the attention to verifying whether

$$
x_{n}^{p} P\left(S_{n} / b_{n} \in x_{n} A\right) \longrightarrow \nu(A),
$$

for much smaller class of sets $A \in \mathcal{B}^{d}$ than the whole ring of bounded away from zero sets of $\nu$-continuity. For example it is enough to consider sets $A_{ \pm}^{y}, y \in \mathbb{R}^{d}$, defined by (18) or "radial" sets described in (11). However, the problem does not seem to be easier after simplification of such kind.

Fortunately, there are methods of essential reduction of (21) to problems depending on properties of joint distributions of a fixed finite number of random variables $X_{1}, X_{2}, \ldots, X_{m}$. These methods were discussed in great detail in Jakubowski (1997) for the case $d=1$. Here we shall describe only basic steps in derivation of their multidimensional versions.

In all considerations the following generalization of the wellknown Bonferroni's inequality is crucial. 
Lemma 5 (Lemma 3.2, Jakubowski, 1997). Let $Z_{1}, Z_{2}, \ldots$ be stationary random vectors taking values in a linear space $\left(E, \mathcal{B}_{E}\right)$. Set $T_{0}=0, T_{m}=\sum_{j=1}^{m} Z_{j}, m \in \mathbb{N}$. If $U \in \mathcal{B}_{E}$ is such that $0 \notin U$, then for every $n \in \mathbb{N}$ and every $k \in \mathbb{N}, k \leq n$, the following inequality holds:

$$
\begin{aligned}
& \left|P\left(T_{n} \in U\right)-n\left(P\left(T_{k+1} \in U\right)-P\left(T_{k} \in U\right)\right)\right| \\
& \quad \leq 3 k P\left(Z_{1} \neq 0\right)+2 \sum_{\substack{1 \leq i<j \leq n \\
j-i>k}} P\left(Z_{i} \neq 0, Z_{j} \neq 0\right) .
\end{aligned}
$$

To be applied effectively, inequality (22) requires that random vectors $Z_{n}$ with great probability take value 0 and that clusters of nonzero values are essentially of short length (i.e. of size $k$ ). This can be achieved by subtracting from $X_{k} / b_{n}$ their truncation around origin (in such a way that the total sum $S_{n} / b_{n}$ is little perturbed - typical property of heavy-tailed random elements), and imposing mixing conditions which guarantee a kind of asymptotic independence of remaining "big" parts of components. The whole procedure is laborious and completely analogous to the one-dimensional case, hence we refer to Jakubowski (1997) for details.

We shall consider three cases of particular interest: $\psi$-mixing, $m$-dependent and $\phi$-mixing sequences (for definitions see Bradley and Bryc, 1985, or Jakubowski, 1993) satisfying the following "usual conditions":

U0. $X_{1}, X_{2}, \ldots$ are strictly stationary random vectors.

U1. $\left\{b_{n}\right\}$ is a $1 / p$-regularly varying sequence for some $p, 0<p<$ 2 .

U2. For some $K_{0}<+\infty$

$$
\sup _{n \in \mathbb{N}} \sup _{x>0} x^{p} \cdot n \cdot P\left(\left\|X_{1}\right\|>x \cdot b_{n}\right) \leq K_{0} .
$$


U3. If $p=1$, then the law of $X_{1}, \mathcal{L}\left(X_{1}\right)$, is symmetric.

U4. If $1<p<2$, then $E X_{1}=0$.

Theorem 6 Suppose $\left\{X_{k}\right\}$ is exponentially $\psi$-mixing (i.e. $\psi(n)$ $\leq K \eta^{n}, n=1,2, \ldots$, for some $K>0$ and $0<\eta<1$ ), and such that

$$
\psi(1)<+\infty .
$$

Then for all $x_{n}$ increasing slowly enough, as $n \rightarrow+\infty$,

$$
x_{n}^{p}\left|P\left(S_{n} / b_{n} \in x_{n} A\right)-n P\left(X_{1} / b_{n} \in x_{n} A\right)\right| \rightarrow 0,
$$

for all $A \in \mathcal{B}^{d}, \bar{A} \not \supset 0$. In particular, $n P\left(X_{1} \in b_{n} \cdot A\right) \rightarrow \nu(A)$ implies

$$
x_{n}^{p} P\left(S_{n} / b_{n} \in x_{n} A\right) \rightarrow \nu(A) .
$$

Theorem 7 Let $\left\{X_{k}\right\}$ be $m$-dependent. Then for all $x_{n}$ increasing slowly enough

$$
\begin{aligned}
x_{n}^{p} \mid P\left(S_{n} / b_{n} \in x_{n} A\right) & (27) \\
-n\left(P\left(S_{m+1} / b_{n} \in x_{n} \cdot A\right)-P\left(S_{m} / b_{n} \in x_{n} \cdot A\right)\right) \mid & \rightarrow 0,
\end{aligned}
$$

for all $A \in \mathcal{B}^{d}, \bar{A} \not \supset 0$. In particular, if

$$
n\left(P\left(S_{m+1} \in b_{n} \cdot A\right)-P\left(S_{m} \in b_{n} \cdot A\right)\right) \rightarrow \nu(A),
$$

as $n \rightarrow+\infty$, then

$$
x_{n}^{p} P\left(S_{n} / b_{n} \in x_{n} A\right) \rightarrow \nu(A) .
$$

Theorem 8 Suppose $\left\{X_{k}\right\}$ is exponentially $\phi$-mixing Then for all $x_{n}$ increasing slowly enough

$$
\begin{array}{r}
\limsup \limsup _{n} x_{n}^{p} \mid P\left(S_{n} / b_{n} \in x_{n} A\right) \\
-n\left(P\left(S_{m+1} / b_{n} \in x_{n} \cdot A\right)-P\left(S_{m} / b_{n} \in x_{n} \cdot A\right)\right) \mid=0,
\end{array}
$$

for all $A \in \mathcal{B}^{d}, \bar{A} \not \supset 0$. In particular, if for each $m \in \mathbb{N}$ we have

$$
n P\left(S_{m} \in b_{n} \cdot A\right) \rightarrow \nu_{m}(A)
$$


and, as $m \rightarrow \infty$,

$$
\nu_{m+1}(A)-\nu_{m}(A) \rightarrow \nu(A)
$$

then

$$
x_{n}^{p} P\left(S_{n} / b_{n} \in x_{n} A\right) \rightarrow \nu(A) .
$$

Remark 9 Theorems 6-8 can be used as tools for proving limit theorems based on Theorems 3 and 4. For example Theorem 6 leads to a result similar to that of Davis (1983) (obtained by purely one-dimensional methods). Theorem 7 allows proving results for $m$-dependent stationary random vectors due to Jakubowski and Kobus (1989) and Kobus (1995) (originally obtained by the point processes technique). Theorem 8 corresponds to Theorem 3.9 in Jakubowski (1997), and gives a counterpart to the early Ibragimov's central limit theorem (Ibragimov, 1962).

Remark 10 Let us notice that in formulas (27), (29) and (33) probabilities of large deviations "in direction" of the set $A$ does not depend on values of random variables outside of the "direction" $A$. This fact is far from being obvious! In particular, in the list of "usual conditions" we did not assume regularity in all "directions" (U2 says that there is no "dominating direction"), and so the whole sum $S_{n} / b_{n}$ may be divergent while Condition $\mathrm{LD}_{d}$ holds for some family of sets $A$.

Remark 11 In Davis and Hsing (1995), under less general conditions, an interesting probabilistic representation is given for constants $c_{+}$and $c_{-}$appearing in Theorems 1 and 2. This representation is expressed in terms of functionals of certain point processes naturally associated with the sequence $\left\{X_{k}\right\}$. Since the structure of the multidimensional limit law is more complicated than in the case $d=1$, it would be interesting to extend Davis and Hsing's results and explain the mechanism of generating the limiting Lévy measure in Condition $\mathrm{LD}_{d}$. 


\section{REFERENCES}

Bradley, R. and Bryc, W., Multilinear forms and measures of dependence between random variables, J. Multivariate Anal., 16 (1985) 335-367.

Davis, R.A., Stable limits for partial sums of dependent random variables, Ann. Probab., 11 (1983) 262-269.

Davis R.A. and Hsing, T., Point processes and partial sum convergence for weakly dependent random variables with infinite variance, Ann. Probab. 23 (1995) 879-917.

Denker, M. and Jakubowski, A., Stable limit distributions for strongly mixing sequences, Stat. Probab. Letters, 8 (1989) 477483.

Ibragimov, I.A., Some limit theorems for stationary processes, Theory Probab. Appl., 7 (1962) 349-382.

Jakubowski, A., Asymptotic Independent Representations for Sums and Order Statistics of Stationary Sequences, Rozprawy, Uniwersytet Mikołaja Kopernika, Torun 1991.

Jakubowski, A., Minimal conditions in $p$-stable limit theorems, Stochastic Process. Appl. 44 (1993) 291-327.

Jakubowski, A., Minimal conditions in $p$-stable limit theorems II, to appear in Stochastic Process. Appl. (1997)

Jakubowski, A. and Kobus, M., $\alpha$-stable limit theorems for sums of dependent random vectors, J. Multivariate Anal., 29 (1989) 219-251.

Jakubowski, A. and Szewczak, Z.S., A normal convergence criterion for strongly mixing stationary sequences, in: Limit Theorems in Probability and Statistics, Pécs 1989, Coll. Math. Soc. J. Bolyai, 57 (1990), 281-292.

Kobus, M., Generalized Poisson distributions as limits of sums for arrays of dependent random vectors, J. Multivariate Anal., 52 (1995) 199-244. 
Samorodnitsky, G. and Taqqu, M.S., Stable Non-Gaussian Random Processes. Stochastic Models with Infinite Variance, Chapman and Hall, London 1994.

Szewczak, Z.S, Large deviation criterion for CLT for strictly stationary sequences, submitted, (1996). 